\title{
New constraints on galactic CRE transport from radio continuum observations
}

\author{
Ralf-Jürgen Dettmar* \\ Ruhr-Universität Bochum, Fakultät für Physik und Astronomie, Astronomisches Institut \\ (AIRUB), Universitätsstrasse 150, 44801 Bochum, Germany \\ E-mail: dettmareastro.rub.de

\section{Volker Heesen} \\ Universität Hamburg, Hamburger Sternwarte, Gojenbergsweg 112, 21029 Hamburg, Germany \\ E-mail: volker.heesen@hs.uni-hamburg.de
}

\section{Arpad Miskolczi}

Ruhr-Universität Bochum, Fakultät für Physik und Astronomie, Astronomisches Institut (AIRUB), Universitätsstrasse 150, 44801 Bochum, Germany

E-mail: Miskolczi@astro.rub.de

\section{Yelena Stein}

Centre de Données astronomiques de Strasbourg, Observatoire de Strasbourg 11, rue de l'Université, 67000 Strasbourg, France

E-mail: yelena.stein@astro.unistras.fr

\section{and the CHANG-ES Team}

Judith Irwin (PI), Dept. of Physics, Engeneering Physics, \& Astronomy, Queen's University, Kingston, Ontario, Canada, K7L $3 N 6$

E-mail: irwinja@queensu.ca

\begin{abstract}
The importance of cosmic rays and magnetic fields for the physics of the interstellar medium and the evolution of galaxies is demonstrated by a number of recent numerical simulations. We present new observations of the cosmic ray electron transport as well as the magnetic field strength and structure in galactic halos from radio-continuum polarization studies of edge-on disk galaxies obtained by the CHANG-ES (Continuum Halos in Nearby Galaxies - an EVLA Survey) project. The observations are discussed in the context of transport models for Cosmic Ray Eelctrons (CREs) from the star-forming disks into the halo. The observations also allow to derive the magentic field structure in galactic halos. In addition, it is demonstrated how recent low frequency observations of edge-on galaxies with LOFAR help to further constrain the problem.
\end{abstract}

36th International Cosmic Ray Conference -ICRC2019-

July 24th - August 1st, 2019

Madison, WI, U.S.A.

${ }^{*}$ Speaker. 


\section{Introduction}

Various feedback mechanisms have been discussed in the context of galaxy formation scenarios (e.g., [1]) in order to explain the observed dependence of the long-term global star formation efficiency in dark matter halos of different mass (e.g., [2], [3]). For the mass range of typical disk galaxies several processes related to star formation are under consideration: besides supernova explosions, stellar winds, and radiation pressure, a number of recent papers discuss the possible importance of magnetic fields and cosmic ray pressure as an additional factor for the global dynamics of the interstellar medium (ISM) as well as for the launching of galactic winds (e.g., [4], [5]). In the following, observations of the synchrotron radiation of cosmic ray electrons (CREs) propagating in the magnetic fields of galactic halos are presented. The recent progress in instrumentation at all large radio facilities allows for a broader wavelength coverage at higher sensitivity and thus these new data provide new constraints for the long standing problem of CRE propagation into halos. The significant progress at the lowest frequencies contributes to the discussion: with LOFAR [6] it is now possible to observe the emission from CREs at lower energies which are less affected by energy losses. They represent an old population that has traveled furthest from the site of origin in the star-forming regions of the mid-plane.

\section{Cosmic ray electron transport into galactic halos}

The polarized and frequency dependent radio-synchrotron radiation of CREs allows us to constrain the magnetic field strength and structure in galaxies. The frequency dependence of the synchrotron intensity also provides information on the transport processes for the CREs (e.g., [7]). This, however, requires that the observed emission is corrected for the frequency dependent contribution of the thermal radio-continuum which is also strongly correlated with star-formation. Considerable progress has been made in this regard due to the availability of infrared data from satellite observations such as Spitzer and WISE. Since dust emission in the ISM is a very good proxy for the star formation rate it can be used to correct for the thermal emission (e.g., [8]). The clean synchrotron emission at various frequencies is then to be used for studying the synchrotron emission by CREs with regard to magnetic field strengths and cosmic ray propagation processes such as diffusion or advection.

In a case study of the southern edge-on spiral galaxies NGC 7090 and NGC 7462 based on Australia Telescope Compact Array (ATCA) data, [9] follow the description for CR transport given by [10]. It is demonstrated that advective and diffusive transport of cosmic ray electrons into the halo can be distinguished by fitting a 1-D cosmic ray transport model to the intensity and spectral profiles perpendicular to the galaxy disc. The transport modes of advection and diffusion result in a characteristic shape of the intensity and spectral index profiles. Advection predicts approximately exponential intensity profiles and linear spectral index profiles while, in contrast, diffusion predicts approximately Gaussian intensity profiles and parabolic spectral index profiles (see Fig. 1 below). This was used for the analysis of archival data from the Very Large Array (VLA) and the Westerbork Synthesis Radio Telescope (WSRT) in [11]. As a results of this larger sample it was concluded that the radio halos of most galaxies studied so far are best described by advective transport models. For these galaxies the bulk velocity of the cosmic ray electrons correlates with 
NGC 891: advection model
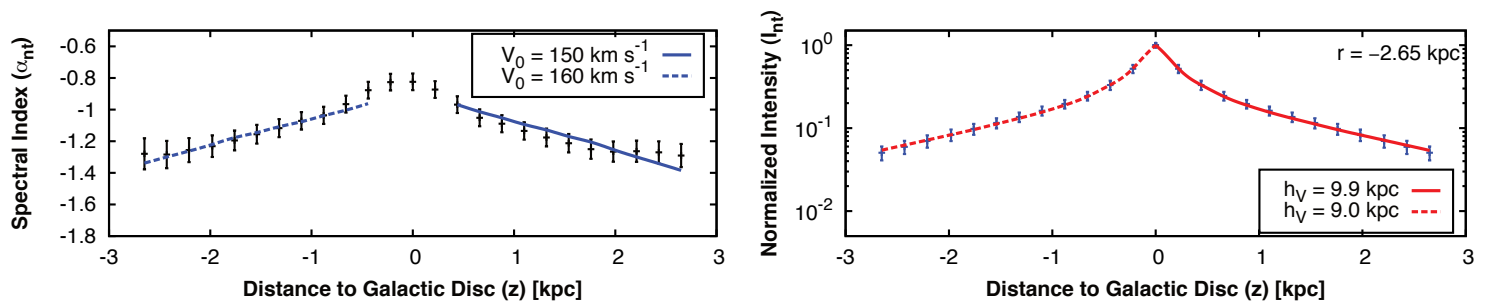

NGC 4565: diffusion model
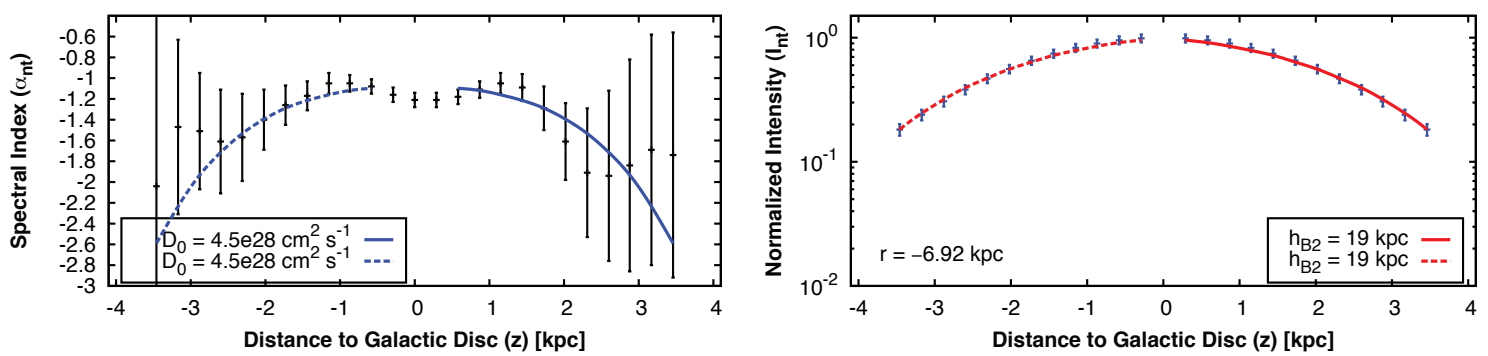

Figure 1: Comparison of advection models in NGC 891 and diffusion models in NGC 4565. In the left panels, data points denote the vertical profile of the non-thermal spectral index between 1.5 and $6 \mathrm{GHz}$ as obtained from the CHANG-ES observations. In the right panels, the data show exponential and Gaussian non-thermal intensity models at $1.5 \mathrm{GHz}$ for NGC 891 and NGC 4565, respectively. Solid lines show the best-fitting cosmic-ray transport models. Advection predicts approximately exponential intensity profiles and linear spectral index profiles; in contrast, diffusion predicts approximately Gaussian intensity profiles and parabolic spectral index profiles. Adopted from [12].

the escape velocity of the galaxies as expected for galactic winds.

\section{First results from CHANG-ES}

This modelling of the synchrotron emission is now to be applied to observations from the Karl G. Jansky Very Large Array (VLA) in the context of the "Continuum HAlos in Nearby Galaxies - an Evla Survey" (CHANG-ES) project. The CHANG-ES sample consists of 35 edge-on spiral galaxies in the local universe selected by angular size and total flux. The targets were observed in the B-, C- and D-array-configurations in C- and L-band [13]. First results for the complete sample have been presented by [14] in combination with a first data release of the D-array data products. This paper also provides an image of an averaged radio-continuum halo or thick disk for the sample. The conclusion that the average edge-on galaxy exhibits an extended radio-continuum halo is different from the finding by [15] claiming that edge-on galaxies do not possess radiocontinuum thick disks or halos that would make the emission look similar to that observed in the Milky Way. The CHANG-ES data now allow for a detailed analysis of the CRE transport and as an example the comparative study of the prototypical edge-on galaxies NGC 891 and NGC 4565 by [12] is shown in Fig. 1. As discussed above, the intensity and spectral index profiles distinguish advection from diffusion dominated transport. Some more general properties of the CHANG-ES 


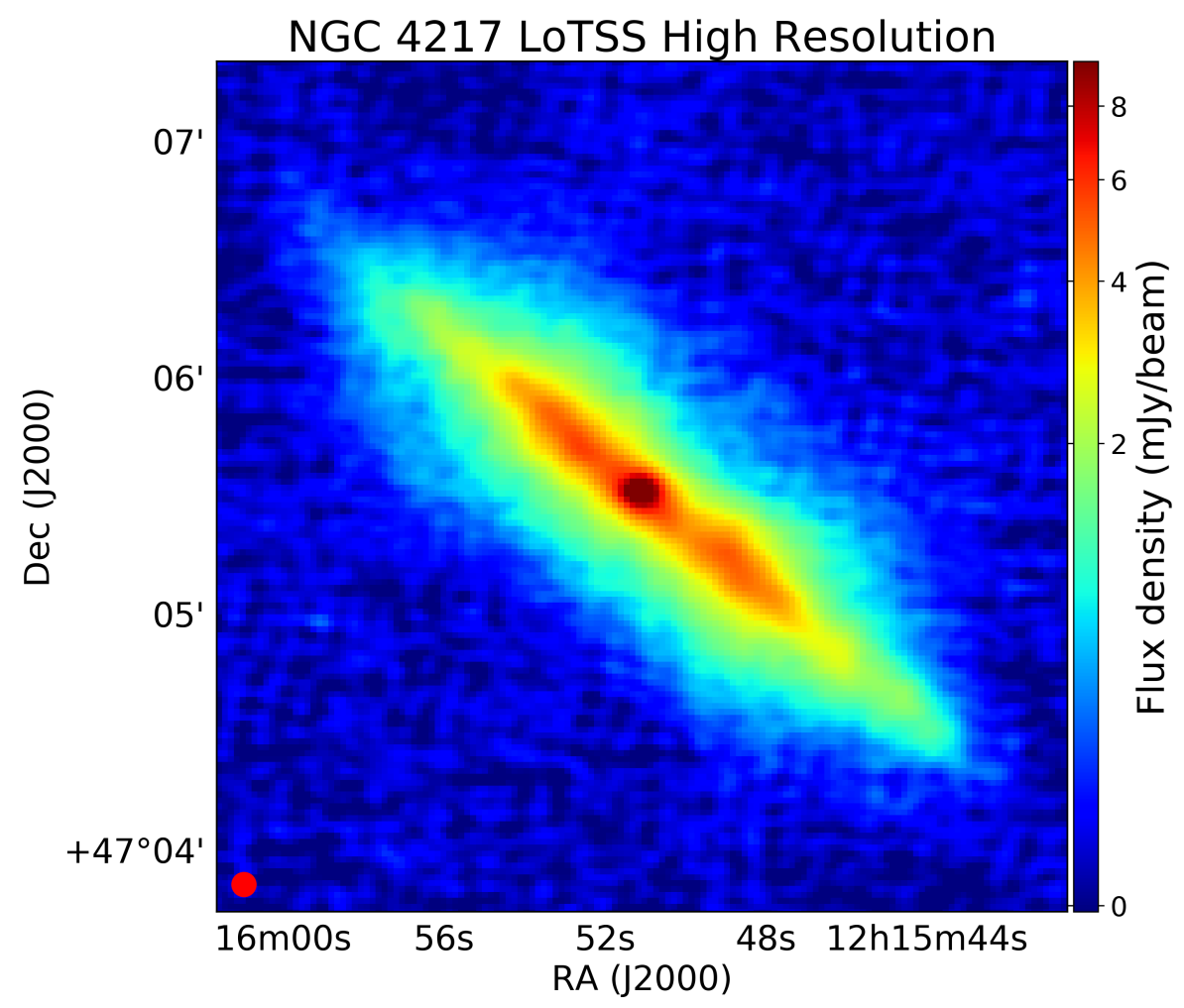

Figure 2: The edge-on galaxy NGC 4217 with a dumbbell-shaped radio-continuum halo is observed by LOFAR at $145 \mathrm{MHz}$ as part of the Data Release 1 of the LoTSS survey ([20], [21]). The reprocessing by A. Miskolczi resulted in the reproduced map with a noise level of $\sim 70 \mu \mathrm{Jy} / \mathrm{beam}$ at 6 arcsec resolution.

sample such as typical scale heights of the radio-continuum disks are provided by [16] and an overview of the CHANG-ES project and of its first results is presented in [17].

\section{Low frequency observations with LOFAR}

Radio-continuum halos of star-forming disk galaxies frequently exhibit a large scale $\mathrm{X}$-shaped magnetic field as, e.g., described for the prototypical case of NGC 5775 in [18]. The extent of the synchrotron emission itself is proof for the presence of a magnetic field reaching far into the halo. Since the low energy cosmic rays are expected to be transported furthest, studies of the corresponding low frequency emission allow us to probe the magnetic field far into the halo. LOFAR now provides observations with unprecedented sensitivity and resolution at such low frequencies as, e.g., demonstrated by the study of NGC 891 [19]. The LOFAR LoTSS survey [20] is now providing a very good base for in-depth studies of many more edge-on galaxies. To demonstrate this potential, Fig. 2 shows a map of NGC 4217 observed as part of the LoTSS Data Release 1 [21].

The dumbbell-shape radio halo (or thick disk) results from the combined effect of a radial decline of the magnetic field strength and the energy losses of the CREs transported into the halo (see [22], [23] for a more detailed discussion). The low-frequency observations of the above mentioned target NGC 4565 with LOFAR presented in a recent paper support the interpretation of a diffusion dominated transport [24] in galaxies with a relatively low star-formation rate. 


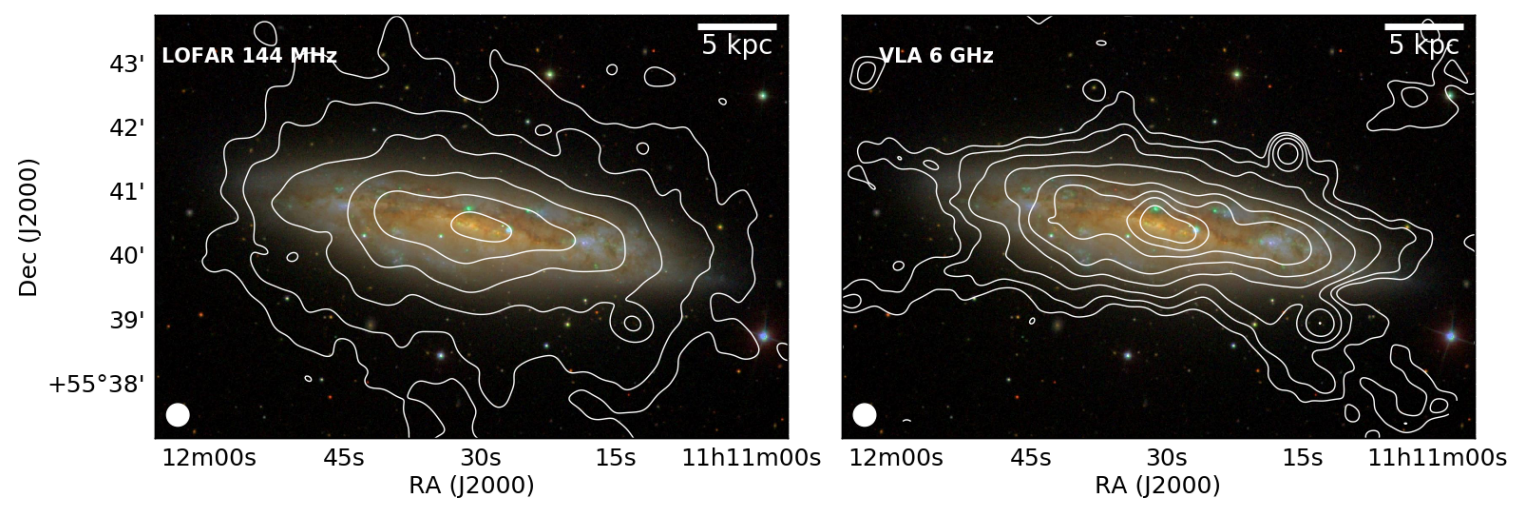

Figure 3: Radio images in contours superimposed on a three-colour optical image from SDSS. Left panel: LOFAR 144-MHz image in contours ranging from $3 \sigma=1.2 \mathrm{mJy}^{-1} \mathrm{beam}^{-1}$ and increasing by a factor of two. The rms noise is 0.4 mJy beam $^{-1}$. Right panel: $4-8-\mathrm{GHz}$ (C band, D array) image in contours starting at $3 \sigma=66 \mu \mathrm{Jybeam}^{-1}$ and increasing by a factor of two. Some CLEANing artefacts are visible in the form of symmetric features on the south side of the galaxy. The rms noise is $22 \mu \mathrm{Jy}_{\text {beam }}{ }^{-1}$. In all panels, the synthesised beam of 21 " FWHM is shown as a filled white circle in the bottom left corner.

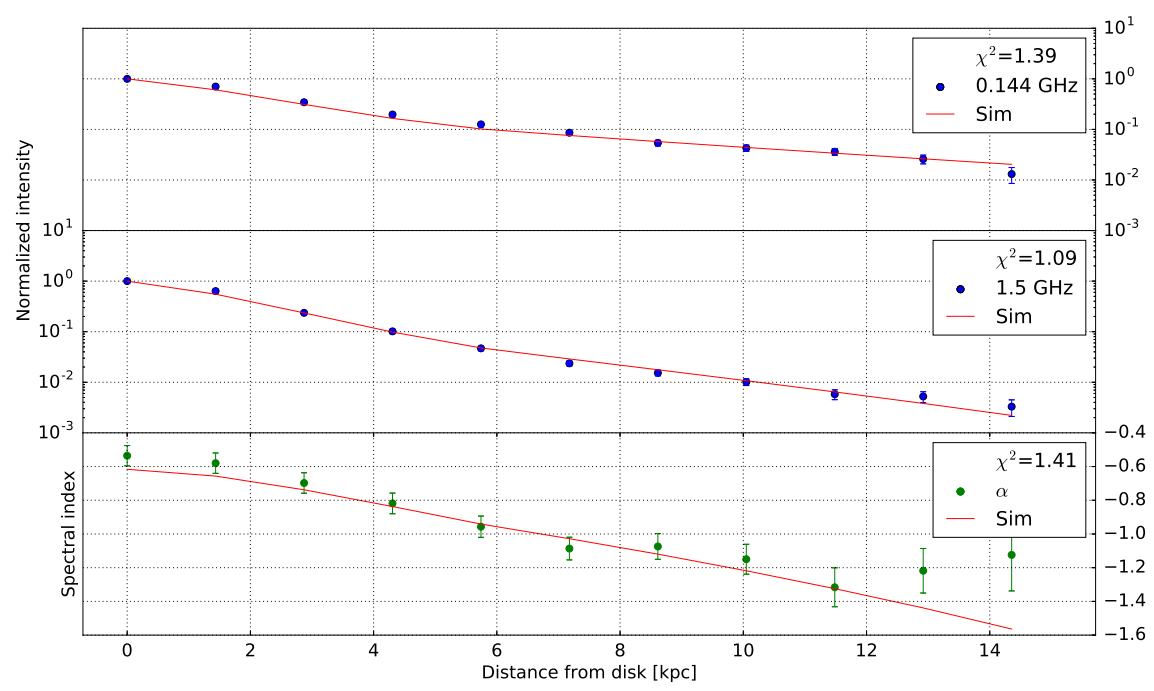

Figure 4: Resulting profiles of the advective cosmic-ray propagation model using a linearly accelerating wind speed. The blue dots represent normalised measured intensities; the top panel shows the LOFAR 144 $\mathrm{MHz}$ measurements and the middle shows the VLA 1.5 GHz measurements. The bottom panel shows the radio spectral index profile. In all panels, the red lines represent the simulated quantity. The scale for the intensity profiles is logarithmic, whereas the spectral index is shown in linear scale.

For several of the CHANG-ES galaxies under study the broad wavelength range between $\mathrm{cm}$ and $m$ wavelength now covered by LOFAR and JVLA observations requires to expand the model. For the case of NGC 3556 the significant larger extend of the radio-halo seen at lower frequencies with LOFAR is shown in comparison to the JVLA observations in Fig. 3. As described by [25], the intensity and spectral index profiles resulting from this combination are better modelled if an increasing advection speed is considered as shown in Fig. 4. In this case advection with a linearly 
increasing wind speed starting at a velocity of $123 \mathrm{~km} \mathrm{~s}^{-1}$ provides the best fit to the measurements at the three frequencies used $(1.5 \mathrm{GHz}, 6 \mathrm{GHz}$, and $144 \mathrm{MHz})$.

\section{Magnetic fields in galactic halos}

Further progress in describing the transport of CRs into galactic halos will certainly also depend on better models of the magnetic field configuration. The observed dumbbell-shape intensity distribution of the radio-continuum emission suggests the wide-spread existence of a X-shaped poloidal halo component of the large scale magnetic field which requires a new generation of magnetic field models as provided, e.g., by [28]. The significance of this poloidal halo component of the magnetic field is supported by a first analyses of the CHANG-ES sample. The wide-band receiver technique at the JVLA allows to apply RM-synthesis reconstruction of the polarized intensity. As an example, Fig. 5 shows the magnetic field structure of the CHANG-ES target NGC 4666 as discussed by [26] in more detail. Besides the prominent halo magnetic field this galaxy is noteworthy since the CHANG-ES data indicate a possible reversal of the magnetic field direction in the plane of the disk.

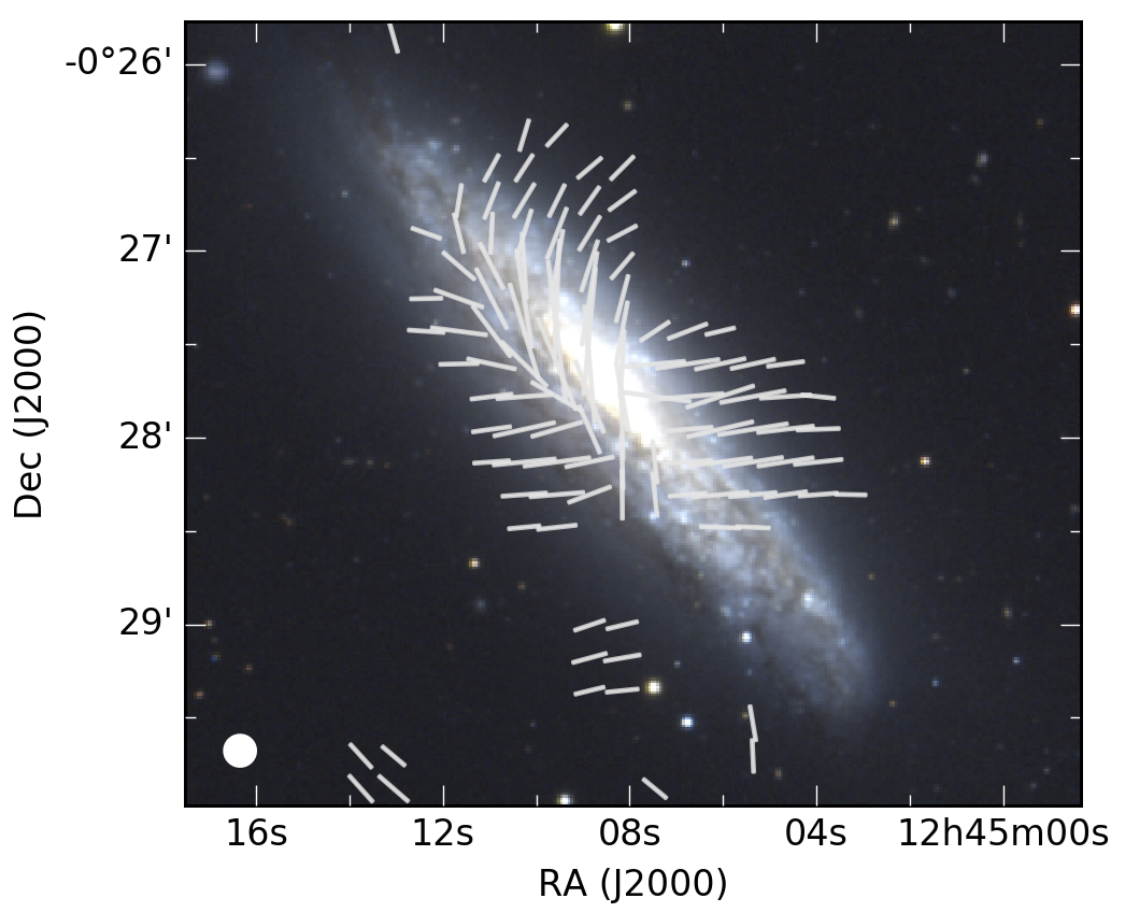

Figure 5: NGC 4666 optical SDSS image produced from the $u, g, r$ filters with apparent magnetic field orientations shown in white with a beam of 10"x10". (adopted from [26]

Yet another recently published case study for a galaxy from the CHANG-ES sample, namely NGC 4013 [27], gives a more complex picture which links the multi-component profile to the starformation history of the galaxy. 


\section{Acknowledgements}

Research at RUB is supported by BMBF Verbundforschung 05A17PC1.

\section{References}

[1] J. Silk, Unleashing Positive Feedback: Linking the Rates of Star Formation, Supermassive Black Hole Accretion, and Outflows in Distant Galaxies, ApJ (2013), 772, 112

[2] P. S. Behroozi et al., The Average Star Formation Histories of Galaxies in Dark Matter Halos from $z$ = 0-8, ApJ (2013), 770, 57

[3] B. P. Moster et al.,Galactic star formation and accretion histories from matching galaxies to dark matter haloes, MNRAS (2013), 428, 3121

[4] P. Girichidis et al., Launching Cosmic-Ray-driven Outflows from the Magnetized Interstellar Medium, ApJL (2016), 816, L19

[5] R. Pakmor et al., Galactic Winds Driven by Isotropic and Anisotropic Cosmic-Ray Diffusion in Disk Galaxies, ApJL (2016), 824, L30

[6] M. P. van Haarlem et al., LOFAR: The LOw-Frequency ARray, A\&A (2013), 556, A2

[7] R. Beck, Magnetic fields in spiral galaxies, A\&A Review (2015), 24, 4

[8] C. J. Vargas et al., CHANG-ES X: Spatially Resolved Separation of Thermal Contribution from Radio Continuum Emission in Edge-on Galaxies, ApJ (2018), 853, 128

[9] V. Heesen et al., Advective and diffusive cosmic ray transport in galactic haloes, MNRAS (2016), 458,332

[10] M. Longair, High Energy Astrophysics, Cambridge University Press

[11] V. Heesen et al., Radio haloes in nearby galaxies modelled with $1 D$ cosmic ray transport using SPINNAKER, MNRAS (2018), 476, 158

[12] P. Schmidt et al., CHANG-ES XVI: An in-depth view of the cosmic-ray transport in the edge-on spiral galaxies NGC 891 and NGC 4565, arXiv [1907.03789]

[13] J. Irwin et al., Continuum Halos in Nearby Galaxies: An EVLA Survey (CHANG-ES). I. Introduction to the Survey, AJ (2012), 144, 43

[14] T. Wiegert et al., CHANG-ES. IV. Radio Continuum Emission of 35 Edge-on Galaxies Observed with the Karl G. Jansky Very Large Array in D Configuration-Data Release 1, AJ (2015), 150, 81

[15] J. Singal et al., Axial Ratio of Edge-On Spiral Galaxies as a Test for Bright Radio Halos, ApJL (2015), 799, L10

[16] M. Krause et al., CHANG-ES. IX. Radio scale heights and scale lengths of a consistent sample of 13 spiral galaxies seen edge-on and their correlations, A\&A (2018), 611, A72

[17] J. Irwin et al., CHANG-ES XVIII: The CHANG-ES Survey and Selected Results, Galaxies (2019) 7, 42

[18] R. Tüllmann et al., The thermal and non-thermal gaseous halo of NGC 5775, A\&A (2000), 364, L36

[19] D. D. Mulcahy et al., Investigation of the cosmic ray population and magnetic field strength in the halo of NGC 891, A\&A (2018), 615, A98

[20] T. W. Shimwell et al., The LOFAR Two-metre Sky Survey. I. Survey description and preliminary data release, A\&A (2017), 598, A104 
[21] T. W. Shimwell, priv. comm

[22] V. Heesen et al., Cosmic rays and the magnetic field in the nearby starburst galaxy NGC 253. I. The distribution and transport of cosmic rays, A\&A (2009a), 494, 563

[23] V. Heesen et al., Cosmic rays and the magnetic field in the nearby starburst galaxy NGC 253. II. The magnetic field structure, A\&A (2009b), 506, 1123

[24] V. Heesen et al., Warped diffusive radio halo around the quiescent spiral edge-on galaxy NGC 4565, arXiv [1907.07076]

[25] A. Miskolczi et al., CHANG-ES XII. A LOFAR and VLA view of the edge-on star-forming galaxy NGC 3556, A\&A (2019), 622, A9

[26] Y. Stein et al., CHANG-ES XIII: Transport processes and the magnetic fields of NGC 4666: indication of a reversing disk magnetic field, A\&A (2019), 623, A33

[27] Y. Stein et al., CHANG-ES XIX: Galaxy NGC 4013 - a diffusion-dominated radio halo with plane-parallel disk and vertical halo magnetic fields, A\&A accepted, arXiv [1906.10650]

[28] K. Ferrière and P. Terral, Analytical models of X-shape magnetic fields in galactic halos, A\&A (2014), 561, A100 